\title{
Conversion of 3D scanned point cloud into a voxel-based representation for crankshaft
}

\section{mass balancing}

\author{
Alexandre Zuquete Guarato ${ }^{\mathrm{a} *}$, Yann Quinsat ${ }^{\mathrm{b}}$, Charyar Mehdi-Souzani $^{\mathrm{b}}$, Claire Lartigue ${ }^{\mathrm{b}}$, Edoardo Sura $^{\mathrm{c}}$ \\ ${ }^{a}$ School of Mechanical Engineering, Federal University of Uberlândia, Uberlândia, Brazil \\ ${ }^{b}$ LURPA, ENS Paris-Saclay, Univ. Paris Sud, Université Paris 13, Sorbonne Paris Cité, Université Paris-Saclay, 94235 Cachan, France \\ ${ }^{c}$ Technocentre, Renault, 78280 Guyancourt, France \\ *Corresponding author. Federal University of Uberlândia. Av. João Naves de Ávila, 2121, CEP 38408-100, Uberlândia, Brazil. Tel.: +55 34 \\ 99273 3537.E-mail: azguarato@ufu.br \\ ORCID: 0000-0001-8428-9646
}

\begin{abstract}
Controlling the part's balance of crankshafts is an important issue for automobile manufacturers. Unbalance measurement is carried out, in general, with high-precision mechanical balancing machines. Each model of crankshaft requires a specialized mechanical balancing machine. We propose a new solution for balancing crankshafts by replacing mechanical measuring systems with a non-contact digitizing system, which allows the acquisition of a representative 3D point cloud of the crankshaft surface. The Voxel2inertia method is proposed to convert 3D scanned point clouds into a voxel-based representation and to calculate the inertia matrix and the unbalance vectors. Theoretical CAD models are used to determinate the best setting for mesh and voxelization. Several levels of error are introduced on CAD models in order to estimate the necessary scanning quality, and thus define the appropriate scanning system for balancing measurements. The Voxel2inertia algorithm is validated on three different crankshaft models that were scanned with the most appropriate digitizing system. The results highlight that this new approach for determining unbalance vectors from scanned data is suitable for replacing the current mechanical measuring systems. This technology can make the production lines more flexible and more adaptable for future changes.
\end{abstract}

Key words: 3D point cloud; 3D scanning; voxel-based representation; STL mesh; crankshaft balancing.

\section{INTRODUCTION}

Crankshafts are parts of the powertrain undergoing high rotations, which require dynamical balancing to reduce vibrations [1]. Generally, crankshaft balancing is performed in various steps. In the first step the reference axis is machined on the forged rough crankshaft. In the second step, the crankshaft unbalance vectors are measured at two reference planes. In the third step, the residual unbalance is corrected by drilling the counterweights. Unbalance measurement is carried out using high-precision mechanical balancing machines. Each measurement device is dedicated to a single model of crankshaft and requires complex and expensive maintenance. Within this context, it could be interesting to find an alternative solution, more flexible and less expensive. A first idea is to replace mechanical measurement systems by a non-contact digitizing system allowing the acquisition of representative data $[2,3]$ of the crankshaft surface. The same measuring system can be used to all the models, and will also allow avoiding the stages of pre-machining and final inspection.

On the other hand, calculating inertia parameters from scanned data is an issue. The crankshaft balancing process requires continuous volumetric representation of the part, whereas digitizing systems deliver discrete data, as a point cloud or mesh for instance, that represents the 
part surface. Most non-contact 3D scanning systems generate non-continuous and partial information of the surface of the object, which can influence the calculations of the inertia matrix.Moreover, 3D scanned data, using non-contact sensors, is generally noisy and incomplete [4-7].This is an issue in the context of crankshaft balancing in which the inertia terms are calculated from a continuous description of the masses of the part. Some authors propose hole filling methods in order to obtain a watertight mesh that is convenient for crankshaft balancing applications [8].

This paper deals with a feasibility study on crankshaft balancing based on data treatment generated by the measurement of the part's surfaces thanks to a contact-less digitizing system. For this purpose, an original method, "Voxel2inertia", is proposed for the calculation of the inertia matrix and unbalance vectors of crankshafts based on discrete data. The discrete data can be acquired by 3D digitizing systems such as stereovision, laser and structured light scanners. The digitized data, in the form of a point cloud, pass through different transformations (mesh, voxelmap) in order to obtain a suitable representation for the inertia calculation (Figure 1).

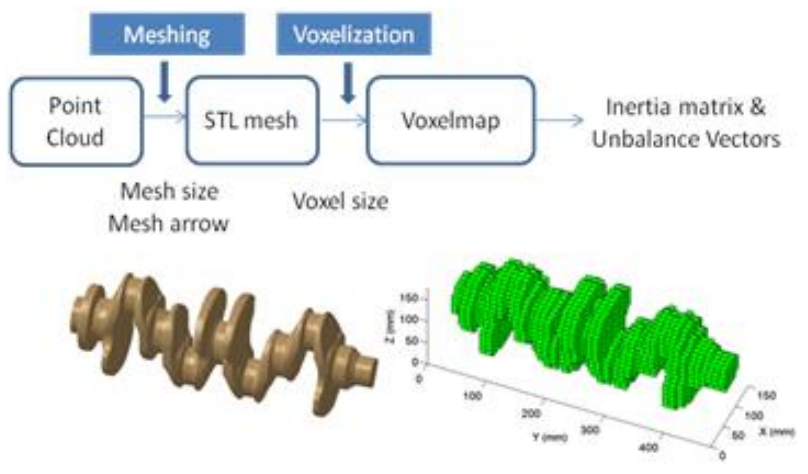

Figure 1.Calculation of unbalance vectors based on scanned data.

The Voxel2inertia method for the calculation of inertia parameters from discrete surface data is developed based on a combination of the classic formulation for computing the terms of inertia and previous work on voxelization methods. The voxel representation of data consists in introducing a volumetric modeling on the cloud of points, so that each voxel will be identified by its position in relation to its neighborhood and the number of points it contains [26].
By this way we could easily identify the inside and outside section of the part and also to take into account the distribution of the masses for the computation of inertial parameters. A sensitivity test of the algorithm is also established in order to reach the necessary quality of scanned data for calculating the crankshaft unbalance vectors. From this sensitivity test, a 3D scanner is selected. The quality of the chosen scanning system will allow an error of less than 5\% in relation to the estimated total mass and reference unbalance vectors. The quality of digitization is estimated by ZuqueteGuarato [9]. Finally, the scanning of three different models of crankshafts allowed validating the Voxel2inertia algorithm.

\section{CALCULATION OF INERTIA PARAMETERS FROM DISCRETE DATA}

Before explaining details about how the parameters of the inertia matrix can be calculated, a review on balancing notations is presented.

\subsection{Balancing notations}

In order to balance a mechanical part in relation to a fixed axis, it is necessary to know its inertia matrix. Unbalanced masses calculation is based on the inertia matrix, which represents the mass distribution of the solid part S. Mass distribution is defined by the gravity centre, moments of inertia and products of inertia.

$$
I_{Q}=\left(\begin{array}{lll}
I_{x x} & I_{x y} & I_{x z} \\
I_{y x} & I_{y y} & I_{y x} \\
I_{z x} & I_{z y} & I_{z z}
\end{array}\right)_{\left(\vec{x}_{1}, \vec{y}_{1}, \vec{z}_{1}\right)}
$$

In automobile industry, the operation of balancing is essentially carried out by measuring the forces or vibrations on two journals of the part. After the unbalance measurement, there is a removal or an addition of mass in order to correct the measured unbalance. In that process, the inertia matrix is not used directly but the concept of unbalance vectors is 
employed. The unbalance vectors are calculated on two predefined reference planes which are orthogonal to the rotation axis. For crankshaft balancing, the rear and the front planes are the reference planes (Figure 2). The unbalance vectors are defined by their norm expressed in g.cm and by their phase (angle between the unbalance vector and the $\mathrm{z}_{1}$ axis) expressed in degrees.

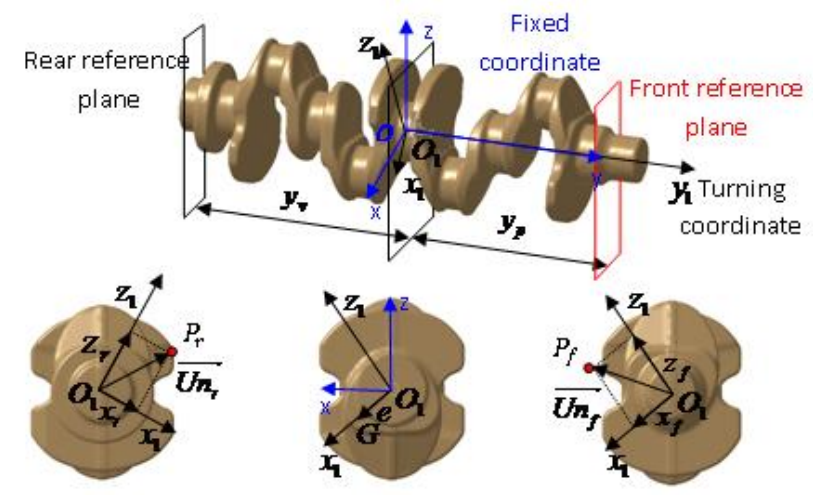

Figure 2.Reference planes and unbalance vectors of the crankshaft.

\subsection{Determination of unbalance vectors}

In the case of crankshaft classic balancing, the two reference planes are located in the extremities of the crankshaft, i.e., they are placed in the midline of the rear (wheel) and front (pinion) journals (Figure 2). The front and rear unbalance vectors $\left(\mathrm{Un}_{\mathrm{r}}, \mathrm{Un}_{\mathrm{f}}\right)$ represent the global distribution of masses in relation to the rotation axis of the mechanical part.

An unbalance vector is positioned at each reference plane. The unbalance vectors can be characterized by concentrated masses $m_{r}$ and $m_{f}$ at the positions $P_{r}\left(x_{r}, y_{r}, z_{r}\right)$ and $P_{f}\left(x_{f}, y_{f}, z_{f}\right), \quad$ respectively. The unbalance vectors are fixed in relation to the coordinate system $\left(\boldsymbol{O}_{1}, \boldsymbol{x}_{\mathbf{1}}, \boldsymbol{y}_{\mathbf{1}}, \boldsymbol{z}_{\mathbf{1}}\right)$ that is rotating with the crankshaft. The original system Unbalanced crankshaft can be expressed by an equivalent system Balanced crankshaft + Rear unbalance + Front unbalance. When a crankshaft is balanced the gravity centre is positioned into the rotation axis (for a static balancing):

$$
M \cdot \boldsymbol{O}_{\mathbf{1}} \boldsymbol{G}=m_{r} \cdot \boldsymbol{O}_{\mathbf{1}} \boldsymbol{U} \boldsymbol{n}_{\boldsymbol{r}}+m_{f} \cdot \boldsymbol{O}_{\mathbf{1}} \boldsymbol{U} \boldsymbol{n}_{\boldsymbol{f}}
$$

The principal inertia axis of a balanced crankshaft is positioned over the rotation axis. Thus, the products of inertia are zero. From the Theorem of Huyghens, the rear and front unbalance vectors become, respectively:

$$
\begin{aligned}
& U n_{r}=m_{r} \sqrt{x_{r}^{2}+z_{r}^{2}}=\sqrt{\left(\frac{I_{x y}-y_{f} \cdot M \cdot e}{Y_{f}-y_{r}}\right)^{2}+\left(\frac{-I_{y z}}{y_{f}-y_{r}}\right)^{2}} \\
& U_{f}=m_{f} \sqrt{x_{f}^{2}+z_{f}^{2}}=\sqrt{\left(\frac{I_{x y}-y_{r} \cdot M \cdot e}{Y_{f}-y_{r}}\right)^{2}+\left(\frac{-I_{y z}}{y_{f}-y_{r}}\right)^{2}}
\end{aligned}
$$

Where e is the radial position of the gravity centre $G$ of the unbalanced crankshaft in coordinate system $\left(\mathrm{O}_{1}, \mathrm{x}_{1}, \mathrm{y}_{1}, \mathrm{z}_{1}\right)$ that is turning with the crankshaft.

For this formulation, the inertia matrix of the solid part Sis required. The inertia matrix represents the mass distribution within the volume of the solid part. However for a balancing process that is based on a $3 \mathrm{D}$ surface data (issued from 3D scanning) the part to be balanced will be known:

- Only by its skin model: 3D digitized data are measured points from the external surface of the part

- In a discrete representation: 3D digitized data are obtained in form of a mesh or a point cloud.

In this way, balancing the crankshaft which was 3D scanned imposes an approximation of necessary integrations to calculate the terms of the inertia matrix. Thus, the determination of unbalance vectors are only possible if a new calculation method of the products of inertia is developed considering the discrete data and its boundaries of integration.

There are few studies in the literature searching to calculate the inertia matrix from discrete data (point cloud or surface mesh, for example). Most of the work concerns the determination of a volume, a surface inertia moment that is based on the skin of the object, a symmetry plane, or geometric principal axes.

Chang and Park [10] propose an algorithm for extracting a plane of symmetry of a point cloud from the determination of the main geometrical axes. They perform an adaptation of the ICP algorithm (Iterative Closest Point) to reduce the computation time by reducing locally the number of points to consider.

Tuzikov, Colliot and Bloch [11] also look for the best symmetry plane from images obtained by magnetic resonance of human brains. In their work, the symmetry plane is also defined from the main geometric axes of inertia.

Liu and Ramania [12] propose an identification of main geometric axes using a principal set of points of the part. Then, the authors gradually add other subsets of points relative to the details of the part in order to improve the results. 
Soldea, Elber and Rivlin [13] propose two exact methods for computing moments of free-form objects. One of them is based on a representation of the boundary of the object with parametric surfaces. In the second method, the boundary is represented by a constant set of trivariate functions, which allows fast reevaluation of the moments.

Crisco and McGovern [14] demonstrate that it is possible to have a correct assessment of the volume, center of gravity and moments of inertia by a slices representation of the studied object. The influence of the number of sections on the quality of the calculation is studied. Nevertheless, this method is not suitable when the input data is only based on the skin of the part because an explicit representation of curves defining the outline of each slice is required. This method is therefore not suitable for data issued from 3D scanning by optical means.

With the objective of finding the position of an object from various 3D-vision sensors, Lichter and Dubowsky [15] propose an interesting approach based on voxels. Authors calculate geometrical inertial parameters (centroid, principal axes, moment matrix) considering that each voxel has an occupancy level proportional to the number of points found within it. For instance, the centroid of the point cloud is calculated as a discrete weighting (linked to the occupancy level) sum of the voxel centres. However, the approach is only dedicated to estimating the pose and the shape of the object in the 3D space, without volume or mass calculations, and cannot be used for unbalance vector estimation. Indeed, the interior of the object is not taken into account in this approach. Nevertheless, the use of voxels to represent the point cloud could be a basis for inertia parameters. In particular, the voxel-space representation defined in [26] allows the calculation of attributes to the voxel in function of the set of points included in the voxel.

This paper proposes to calculate the inertia terms and the unbalance vectors by using a volumetric representation of the part in the form of a set of voxels. The required continuous solid part is approximated by a distribution of concentrated masses. One concentrated mass is located at the barycenter of each voxel.

\section{VOXEL2INERTIA: AN ALGORITHM FOR CALCULATION OF UNBALANCE VECTORS AND CRANKSHAFT BALANCING}

A voxel can be defined by a 3D pixel and generally it has equal length sides, i.e., a cubic shape. In the context of 3D digitization, voxels can be constructed based on the external geometry of the 3D scanned surface. Accordingly to Jones et al. [16], each voxel can be associated to a group of digitized points which represents the skin of the part. Classically the voxels that are based on a point cloud can be classified in two types, [17]:

- The empty voxels that do not contain digitized points;

- The filled voxels that contain a group of points

$\mathrm{M}_{\mathrm{i}}$ from the total point cloud.

This classification is not ideal for the calculation of terms of the inertia. In order to calculate the unbalance vectors it is required to distinguish the voxels that are inside the surface of the part from those outside the surface of the part. Voxel2inertia is an improved inertia calculation algorithm that employs the voxelization method proposed by Patil and Ravi [18]. The algorithm requires a watertight closed STL mesh. If the mesh is not watertight close, the method of managing the intersections is not capable of defining inside and outside voxels. As meshes with holes and inconsistencies are very common in the $3 \mathrm{D}$ digitizing field, some post-scanning treatment is necessary.

The voxelization algorithm constructs a $3 \mathrm{D}$ structure of voxels (voxelmap) based on a STL mesh of the part (Figure 3) and detects the voxels that are inside the skin of the part.

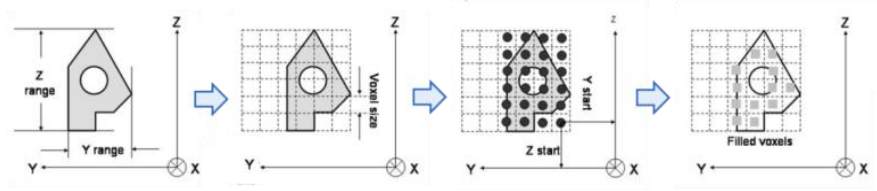

Figure 3.Voxelization of a part section and determination of filled voxels [18].

The set of voxels is based on the coordinate system that is fixed to the part. Hence, the voxel edges are parallel to the axes of the coordinate system of the part. One vertex of the voxels corresponds to the smallest 3D coordinates in each direction of the coordinate system (X, Y and $\mathrm{Z}$ ). The basic principle of the voxelization 
method consists in calculating the intersections between the input STL mesh and a group of projected lines that are parallel with respect to the coordinate system axes and passes through the center of the voxels. In a simple way, voxels that are located between two intersections are considered to be inside of the analyzed object. The intersection process is repeated in the two other directions of the coordinate system in order to refine the voxelmap representation and improve the detection of thin walls. In this way, the size of the voxels has an important impact on the representation of the volume of the part. The algorithm is also sensitive to the chosen size of the voxels for representing the part. As a matter of fact, a set of too large voxels may not detect thin walls.

The voxel representation of the volume of the part can change in function of the voxels size. Reducing the voxels size allows a better representation of the curves and geometry of the part, which is materialized by the STL mesh, and thus, avoids the errors on the mass calculation. In other hand, for a very big voxel size in relation to the volume of the part, some geometric details of the part are neglected.

After the construction of the voxelmap representation of the part and the detection of inside and outside voxels, a $\beta$ index is associated to each voxel. For $\beta=1$ the voxel is inside the part and for $\beta=0$, the voxel is outside the part. A concentrated mass $\Delta \mathrm{m}$ is calculated for each inside voxel as follows:

$$
\Delta \mathrm{m}=\rho \mathrm{V}
$$

Where $\mathrm{V}$ is the volume of the voxel and $\rho$ is the specific mass of the voxel.Surface voxels, i.e., those enclosing at least on face of the initial STL mesh and having at least one exposed voxel face (without a neighbor voxel), are identified. Surface voxels are classified as empty voxels and they are excluded from the list of inside voxels. The identification of surface voxels is performed iteratively in each direction of the projected lines until the final voxelization that has only inside voxels (Figure 4).

Considering that in this balancing application the crankshaft is manufactured on forged steel, it is reasonable to use the hypothesis that the mean specific mass $\rho$ is homogenous across all the part. A 3D structure of voxel barycenters is built. Each barycenter point concentrates the total mass of the voxel. The voxels are cubic and they have edges measuring $\mathrm{S}_{\mathrm{y}}$. The calculation of the terms of the inertia matrix is based on discrete 3D digitized points and only the inside voxels of the STL mesh are considered. The terms of the inertia matrix are calculated with the Eqs. 7 to 12:

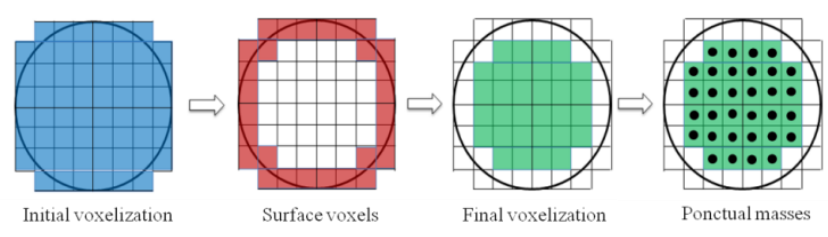

Figure 4: Identification of surface voxels and construction of the final voxelization of a sphere.

Where $x_{i}, y_{i}, z_{i}$ are the barycenter coordinates of the voxels, $\beta$ is the index which is equal to 1 for inside voxels and 0 for outside voxels, and $\Delta m=\rho V$, where $V$ is the volume of the voxel. The components of the inertia matrix are calculated by the Eqs. 7 to 12, as well as the coordinates of the gravity center $G$. The unbalance vectors are calculated thanks to the Equations 4 and 5.

$$
\begin{gathered}
\mathrm{I}_{\mathrm{xx}}=\sum_{\mathrm{V}} \Delta \mathrm{m} \cdot \beta_{\mathrm{i}} \cdot\left(\mathrm{y}_{\mathrm{i}}^{2}+\mathrm{z}_{\mathrm{i}}^{2}\right) \\
\mathrm{I}_{y y}=\sum_{V} \Delta m \cdot \beta_{i} \cdot\left(x_{i}^{2}+z_{i}^{2}\right) \\
\mathrm{I}_{z z}=\sum_{V} \Delta m \cdot \beta_{i} \cdot\left(x_{i}^{2}+y_{i}^{2}\right) \\
I_{x y}=\sum_{V} \Delta m \cdot \beta_{i} \cdot\left(x_{i} \cdot y_{i}\right) \\
\mathrm{I}_{\mathrm{xz}}=\sum_{\mathrm{V}} \Delta \mathrm{m} \cdot \beta_{\mathrm{i}} \cdot\left(\mathrm{x}_{\mathrm{i}} \cdot \mathrm{z}_{\mathrm{i}}\right) \\
\mathrm{I}_{\mathrm{yz}}=\sum_{\mathrm{V}} \Delta \mathrm{m} \cdot \beta_{\mathrm{i}} \cdot\left(\mathrm{y}_{\mathrm{i}} \cdot \mathrm{z}_{\mathrm{i}}\right)
\end{gathered}
$$

The Voxel2inertia calculation algorithm is developed with $M A T L A B^{\circledR}$. It uses the principal steps presented on the Figure 5: read the STL mesh, initial voxelization based on the STL mesh, identification of inside voxels, construction of a concentrated mass structure, calculation of the terms of inertia and the unbalance vectors. Each one of the steps is based on a specific representation (point cloud, mesh and voxelmap) that induces an approximation in relation to the real part. Therefore it is important to evaluate the quality of each 
one of these representations in relation with the analytical calculation (exact calculation).

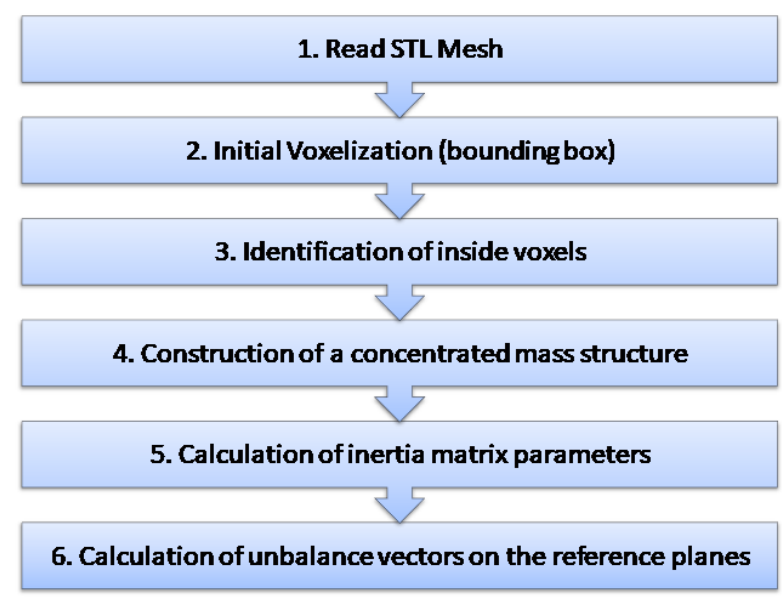

Figure 5.Voxel2inertia algorithm.

A sensitivity analysis is presented in the next section to determine the minimum required quality of digitized data with respect to actual unbalance measurement tolerances. The selection of the suitable 3D scanning sensors is carried out based on the sensitivity analysis.

\section{SENSITIVITY OF THE VOXEL2INERTIA TO REAL DATA AND SELECTION OF THE DIGITIZING SENSOR}

\subsection{Simulation of real digitizing errors on theoretical meshes}

The Voxelization algorithm is tested using theoretical meshes that are extracted from the CAD model of the crankshaft. First of all, the influence of the mesh settings and the size of the voxel are verified. The complete sensitivity tests are presented on [19].

To analyze the sensitivity of the Voxel2inertia algorithm in relation to real digitized data, some defects are introduced into the theoretical data. Noise and trueness variations are simulated by introducing Gaussian noise and scale factors, respectively. Several noise levels and scale factors are tested with the purpose of determining the minimum required digitizing quality allowing inertia parameter and unbalanced vector calculations to be consistent with balancing requirements.

A Gaussian noise is added to the theoretical point cloud derived from the CAD crankshaft model. Five levels of noise are analyzed: $200 \mu \mathrm{m}, 300 \mu \mathrm{m}, 400 \mu \mathrm{m}$, $500 \mu \mathrm{m}$ and $600 \mu \mathrm{m}$. The noise levels are distributed into the three directions $\mathrm{X}, \mathrm{Y}$ and $\mathrm{Z}$. This simulates equivalent digitizing system levels of noise $(6 \sigma)$ of: $33 \mu \mathrm{m}, 50 \mu \mathrm{m}, 66 \mu \mathrm{m}, 83 \mu \mathrm{m}$ and $100 \mu \mathrm{m}$, respectively. These noise levels are consistent to real 3D scanning systems [20].

Each noise level is applied to 10 meshes. In this way 50 meshes are generated from the CAD model. The unbalance vectors are calculated for 10 meshes for each noise level. The maximum tolerated error for respecting the balancing requirements is 5\%. Considering this industrial requirement, the noise level must be lesser than $0.5 \mathrm{~mm}$, which means that the digitizing systems must have an equivalent noise level $(6 \sigma)$ under $83 \mu \mathrm{m}$.

In this work, trueness is supposed to act as a dilatation factor of the digitized data in relation to the reference CAD model. Therefore, a scale factor can stand for relative trueness. Various levels of trueness are tested thanks to different scale factors.

Table 1 and Table 2 present the maximum error for each simulated noise level and scale factor. Results highlight the influence of the noise on the mechanical inertia calculation. Moreover, scale factors between 0.99 and 1.01 respect the balancing tolerance.

Table 1: Unbalance error in relation to noise level.

\begin{tabular}{llllll}
\hline $\begin{array}{l}\text { Noise level } \\
(\mathrm{mm})\end{array}$ & 0.2 & 0.3 & 0.4 & 0.5 & 0.6 \\
\hline $\begin{array}{l}\text { Unbalance } \\
\text { error }(\%)\end{array}$ & 3.01 & 4.25 & 3.17 & 3.34 & 5.97 \\
\hline
\end{tabular}

Table 2: Unbalance error in relation to scale factor.

\begin{tabular}{lccccccc}
\hline $\begin{array}{l}\text { Scale } \\
\text { Factor }\end{array}$ & 0.97 & 0.98 & 0.99 & 1.00 & 1.01 & 1.02 & 1.03 \\
\hline $\begin{array}{l}\text { Unbalance } \\
\text { error }(\%)\end{array}$ & 9.93 & 5.85 & 0.66 & 1.76 & 1.16 & 15.77 & 12.19 \\
\hline
\end{tabular}

To summarize, digitizing systems for which the noise is under $83 \mu \mathrm{m}$ and for which the relative trueness is under $1 \%$ are admissible for crankshaft measurement within the context of contactless balancing. The selection of the appropriate digitizing systems is carried out based on these admissible values of relative trueness 
and noise.In this sensitivity analysis, it is considered that the quality of the current sensors allows a sufficiently high point density not to introduce skew on the determination of unbalance. This assumption is consistent given the voxel calculation method used.Additional method for digitization hole management could be considered in the case of data that are locally too deficient as developed in [8].

\subsection{Selection of a suitable scanning system}

For choosing the suitable scanning systems for an application it is necessary to evaluate their quality of measurement. Several researchers had studied the sources of inaccuracy on 3D scanning systems and how to evaluate them [4-6, 9].

More recently, Mehdi-Souzani et al. [19] proposed a knowledge database of qualified digitizing systems for the selection of the best system according to the application. Based on that work the 3D scanning system GOM Atos III is chosen to be used on the validation of the Voxel2inertia algorithm. The selected system is based on a structured light measurement principle that projects patterns of blue light fringes. Blue light is less sensitive to environment light changes that can lead to measurement errors. This scanning system has, on average, $3.240 \mu \mathrm{m}$ of noise and $0.339 \%$ of relative trueness, which are lesser than the admissible maximum of $83 \mu \mathrm{m}$ for noise and $1 \%$ for relative trueness.

The next section is dedicated to the validation of the Voxel2inertia algorithm that is carried out with crankshafts from Renault SA.

\section{VALIDATION OF VOXEL2INERTIA ALGORITHM BASED ON CRANKSHAFT DIGITIZING}

The validation process of the Voxel2inertia method is based on 3D scanned data of three forged crankshafts whose imbalance vectors were previously measured in a mechanical balancing machine. The crankshafts were furnished by Renault automation from a production line in France.

The balancing process is carried out on Stations 1, 2 and 3 of the production line. Station 1 consists on the pre-machining of rotation axes of the rough forged crankshafts. Station 2 dynamically measures the unbalance vectors of the crankshafts. On the Station 3, the reference axis of rotation is drilled. This operation creates a lack of material within the skin envelope of the crankshafts that would impact the unbalance calculation with the Voxel2inertia algorithm. In order to avoid these errors the crankshafts are taken out the line after passing through the Stations 1 and 2 and before the Station 3.After passing through Stations 1 and 2, the outer surfaces of the crankshafts are mainly on rough forging with the exception of the cylindrical machined surfaces made on the extremities of the crankshafts.

Three different models of crankshafts from fourcylinder diesel engines and a gasoline engine were used (Figure 6). Tests are carried out on 1 crankshaft of each typology. These crankshafts are chosen with the aim of validating the efficiency and flexibility of the Voxel2inertia algorithm for working on different parts.

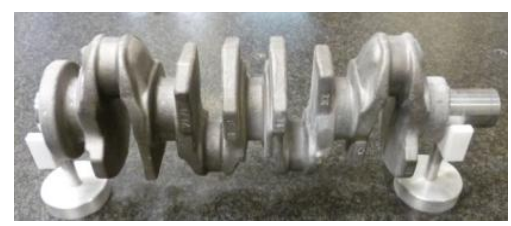

a)

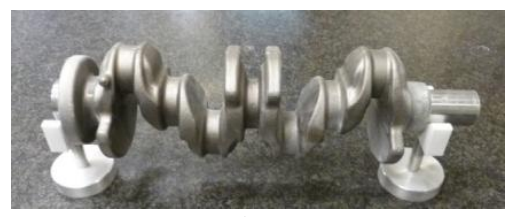

b)

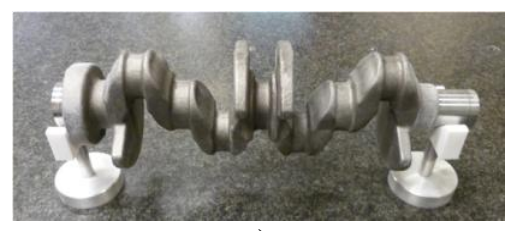

c)

Figure 6: Crankshafts a) Diesel 1, b) Diesel 2 and c) Gasoline 1 chosen for the Voxel2inertia validation.

The validation of Voxel2inertia algorithm for calculating unbalance vectors based on 3D scanned data is performed in two steps. In the first part, crankshafts are 3D scanned with the chosen system and scanning results are presented. In the second part, the unbalance vectors are calculated based on the digitized crankshafts.

In order to obtain the same conditions for scanning, the Voxel2scan path planning method [21] is applied to the selected digitizing system. Voxel2scan algorithm is an original approach to generate a path planning for scanning objects with any geometry and with any type of 
3D scanning optical sensors. This method is based on the voxelization of a reference mesh of the object. The size of the voxel is defined according to the sensor Field of View (FOV). To each voxel, a unique point of view is associated in function of visibility and digitizing quality criteria. Whatever the sensor, the method provides the minimum set of admissible points of view to ensure the surface digitizing with a given quality.

The scanning methodology is defined by a set of points of view determined by the Voxel2scan method [21]. Each point of view is defined by a position and an orientation in the space in which the scanner has to be placed in order to digitize the maximum surface of the object and to minimize the measurement time. After defining the positions and orientations, i.e. the points of view, for placing the scanner in relation to the crankshaft, the digitizing process is performed. For each used point of view, the scanning system delivers a mesh that represents the measured geometry. The final result is a fusion of several meshes. For each crankshaft, the time of scanning is around 16 minutes. This is due to the non-automated displacement system of the Atos III system: a tripod.

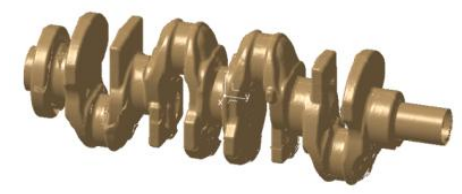

a)

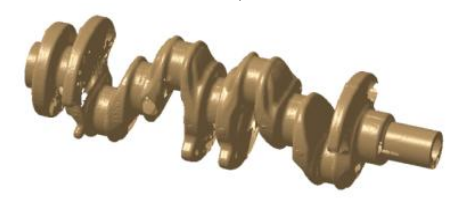

b)

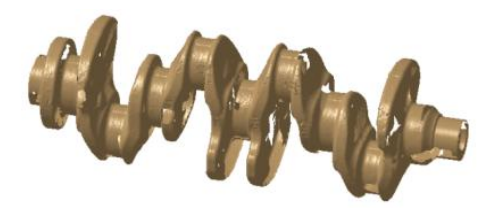

c)

Figure 7: Crankshafts a) Diesel 1, b) Diesel 2 and c) Gasoline 1 chosen for the Voxel2inertia validation.

The scanning results are presented on Figure 7. It is interesting to note that there are scanning holes mostly on the zones that are hard to access such as the surfaces between the central counterweights and the linking arms between the cylindrical surfaces. The voxel2scan method can identify these difficult to scan zones before the scanning process. Other scanning holes also appear on the extremities of the crankshafts near the axis of rotation. This is due to the cylindrical artifacts with positioning targets that were assembled to the extremities of the parts for allowing the registering of the meshes on the same coordinate system.

As stated before, Voxel2inertia algorithm requires a watertight closed triangular mesh. Therefore, the scanned data must be post-processed so that it can exploitable in the Voxel2inertia algorithm.

The first post-treatment consists in the hole filling of the meshes, which in this case, was made by the function Fill holes of the Digitized Shape Editor from Catia V5() software (Figure 8). Then, following the ideal settings [19], the function remesh is used for a mesh size of $S_{m}=1 \mathrm{~mm}$ and for a mesh arrow of $S_{a}=0.06 \mathrm{~mm}$.

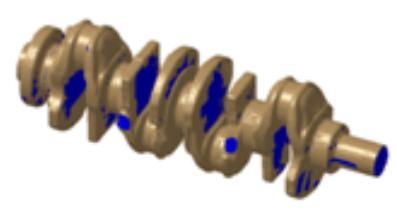

Figure 8: Crankshaft with scanning holes (in brown) and after hole filling (in blue).

In order to calculate the unbalance vectors, the coordinate system of the mesh should match the calculation coordinate system of Voxel2inertia method. Thus, some canonical structures are associated to parts of the mesh by the minimum least squares criteria. Two planes (XZ and YZ) are created on the symmetric region of the crankshaft and two cylinders are associated to the extremities of the crankshaft (Figure 9). These references recreate a geometrical axis of the crankshaft that allows its positioning with respect to the calculation coordinate system. Plane and cylinder associations are performed using Quick Surface Reconstruction workbench and the registration is realized on Digitized Shape Editor workbench with the tool "align with constraints" of Catia V5@ software.

This operation of defining a geometrical axis for repositioning the crankshafts allows the Voxel2inertia method to calculate the unbalance vectors in the same coordinate system that is used on the mechanical balancing machines. The next section presents the 
calculation results of the unbalance vectors for the three models of crankshaft.

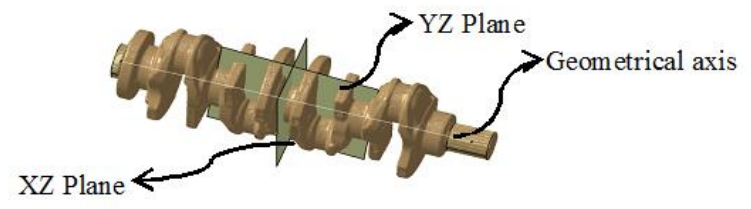

Figure 9: Definition a geometrical axis through planes and cylinders references.

\section{UNBALANCE CALCULATION RESULTS AND DISCUSSION}

The results of unbalance vectors that were measured by the mechanical balancing machines as well as the unbalance vectors obtained from the Voxel2inertia method based on 3D scanned data are presented on the Table 3 and Figures 10 and 11.

Table 3: Comparison of the unbalance vectors obtained from the balancing machine and from the Voxel2inertia method for 3 models of crankshaft.

\begin{tabular}{llll}
\hline $\begin{array}{l}\text { Crankshaft } \\
\text { model }\end{array}$ & $\begin{array}{l}\text { Calculation } \\
\text { method }\end{array}$ & $\begin{array}{l}\text { Rear } \\
\text { unbalance } \\
\left(10^{-3} \mathrm{Kg} . \mathrm{m}\right)\end{array}$ & $\begin{array}{l}\text { Front } \\
\text { unbalance } \\
\left(10^{-3} \mathrm{Kg} . \mathrm{m}\right)\end{array}$ \\
\hline Diesel 1 & Balancing machine & 2.87 & 3.35 \\
& $\begin{array}{l}\text { Scanning }+ \\
\text { Voxel2inertia }\end{array}$ & 2.75 & 3.26 \\
& Error (\%) & 4.24 & 2.84 \\
\hline Diesel 2 & Balancing machine & 2.40 & 1.58 \\
& $\begin{array}{l}\text { Scanning + } \\
\text { Voxel2inertia }\end{array}$ & 2.45 & 1.63 \\
& Error (\%) & 1.81 & 3.56 \\
\hline Gasoline 1 & Balancing machine & 1.75 & 0.74 \\
& $\begin{array}{l}\text { Scanning + } \\
\text { Voxel2inertia }\end{array}$ & 1.82 & 0.75 \\
& Error (\%) & 3.87 & 1.44 \\
\hline
\end{tabular}

The presented results are the average of 10 calculations for each crankshaft. It is important to highlight that no deviations are noticed on 10 calculations results for each crankshaft. Indeed, the Voxel2inertia method always constructs the voxelmap starting from the same references, which do not change for the same mesh.

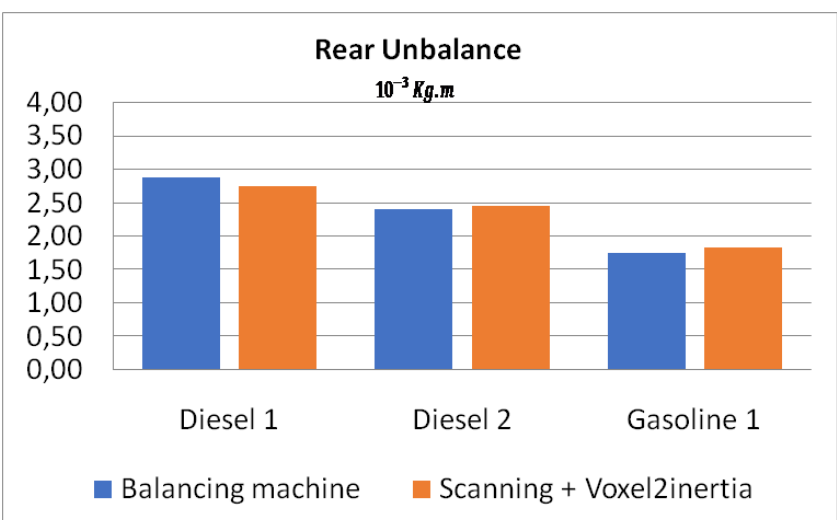

Figure 10: Rear unbalance for the three models of crankshaft.

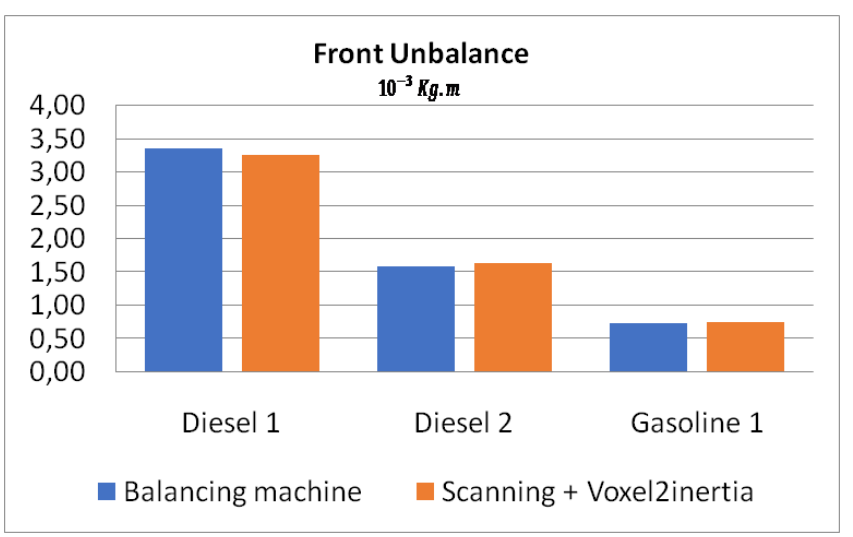

Figure 11: Front unbalance for the three models of crankshaft

All the meshes have been set to a maximum mesh size of $\mathrm{S}_{\mathrm{m}}=1 \mathrm{~mm}$ and a maximum mesh arrow of $\mathrm{S}_{\mathrm{a}}=$ $0.06 \mathrm{~mm}$. Due to the different volumes of each crankshaft, voxel sizes are set accordingly. The voxel sizes used are $S_{\mathrm{V}}=0.65 \mathrm{~mm}, \mathrm{~S}_{\mathrm{V}}=0.60 \mathrm{~mm}$ and $\mathrm{S}_{\mathrm{V}}=0.55 \mathrm{~mm}$ for the crankshaft models Diesel 1, Diesel 2 and Gasoline 1, respectively [7].

The results highlight that the unbalance vectors calculated with Voxel2inertia method from scanned data using the scanning system Atos III are similar to the unbalance vectors measured by the mechanical balancing machine. The average error is $2.96 \%$, which is below the $5 \%$ maximum error required for the crankshaft production line of Renault. These results 
confirm that even though the post-treatments are manually made with Catia V5(C) software, they are appropriated for filling the holes and for repositioning the meshes into the coordinate system of Voxel2inertia method. A recent study applies a morphing-based that relies on the CAD model for filling digitizing holes. This hole filling method preserves the original curvature of the scanned mesh and allows a reduction of the sensor orientations during the digitizing process [8]. Thus, a complete scanning is no longer necessary and the overall unbalance process time is reduced.

The Voxel2inertia algorithm for calculating the unbalance vectors, coupled with Atos III scanning system, has a great potential for replacing the current mechanical balancing machines. This method can be used on parts with different geometry and with different types of scanning sensors, allowing the same production line to manufacture different types of crankshaft in the future.

\section{CONCLUSIONS}

Crankshafts are generally balanced with high precision mechanical balancing machines on industry. Each model of crankshaft requires a specific balancing machine. In order to make the production line more flexible and adaptable to production changes, this paper presents a new method for balancing by determining the unbalance vectors from a 3D representative scanned data. In this way the specialized mechanical balancing systems can be replaced by one 3D scanner for the whole process. The Voxel2inertia method was first tested through simulations based on the nominal CAD models for estimating the necessary quality of scanning and for defining the appropriate digitizing system for crankshaft balancing. Three crankshafts, previously measured by a mechanical balancing system, were used to validate the Voxel2inertia algorithm.

The procedure of scanning is carried out following the points of view that are obtained from the Voxel2scan method in order to have the same digitizing conditions for all parts. All error estimations on the unbalance vectors with the Voxel2inertia method in relation to the mechanical measurement are under the $5 \%$ maximum error required by the production plant of Renault. The average error is $2.96 \%$, which proves that this methodology is suitable for replacing the current mechanical measuring machines.
The Voxel2inerita calculation method, associated with the 3D scanner, can be used on different types of crankshafts. This allows the productions lines to be more flexible and more adaptable to production changes. Also, the scanners are generally cheaper than the specialized mechanical measurement machines.

Future work relies on integrating the post-treatments into the Voxel2inertia algorithm for automating all the process from the 3D scanning to the calculation of unbalance vectors. Hole filling methods [8] can be used improve the scanning path planning because a complete scanning process is no longer necessary. Only strategic regions of the crankshaft are scanned with fewer sensor positions and a CAD based mesh is deformed over the scanned mesh for filling the holes.

A dedicated displacement system should be conceived specially for the chosen sensor based on the Voxel2scan method. These improvements can lead to a faster and more robust crankshaft balancing for the production line than the classic ones.

This new balancing method could be also used on other parts or machines such as rotary engines [23-25].

\section{ACKNOWLEDGEMENTS}

This work was supported by Renault SA and CAPES und Grant Science without Borders Program- CSF-PAJT - 88887.068777/2014-00.

\section{REFERENCES}

1. Wowk V (1998) Machinery vibration: balancing. McGraw-Hill Professional, USA

2. Charron JL (2004) Mesure sans Contact: Méthodes Optiques (partie 1) [Contaclessmeasurement (part 1)] Techniques de l'ingénieur: Mesures et Contrôle R1332 V1. http://www.techniques-ingenieur.fr/basedocumentaire/mesures-analyses-th $1 /$ mesures-delongueurs-et-d-angles-42408210/mesures-sanscontact-r1332/

3. Charron JL (2004) Mesure sans Contact: Méthodes Optiques (partie 2) [Contacless measurement (part 2)] Techniques de l'ingénieur: Mesures et Contrôle, R1333 V1. http://www.techniques-ingenieur.fr/basedocumentaire/mesures-analyses-th $1 /$ mesures-de- 
longueurs-et-d-angles-42408210/mesures-sanscontact-r1333/

4. Barbero BR, Ureta ES (2011) Comparative study of different digitization techniques and their accuracy. Computer-Aided Design 43.2: 188-206.

5. Mahmud M, Joannic D, Roy M, Isheil A, Fontaine JF (2011) 3D part inspection path planning of laser scanner control on the uncertainty. Computer-Aided Design 43(4): 345-355

6. Audfray N, Mehdi-Souzani C, Lartigue C (2012) Assistance to automatic digitizing system selection for 3d part inspection. ASME 2012 11th Biennial Conference on Engineering Systems Design and Analysis, American Society of Mechanical Engineers,8231, Nantes, July 2-4

7. Zuquete-Guarato A (2013) Métrologie 3D de pièces de formes complexes par moyens optiques: une application à l'equilibrage de vilebrequins. $\mathrm{PhD}$ dissertation. Ecole Normale Supérieure de Cachan

8. Quinsat Y, Lartigue C (2015) Filling holes in digitized point cloud using a morphing-based approach to preserve volume characteristics. The Int J Adv Manuf Technology 81.1-4:411-421. doi:10.1007/s00170-015-7185-0

9. Zuquete-Guarato A, Mehdi-Souzani C, Quinsat Y, Lartigue C, Sabri L (2012) Towards a new concept of in-line crankshaft balancing by contactless measurement: process for selecting the best digitizing system. ASME $2012 \quad 11^{\text {th }}$ Biennial Conference on Engineering Systems and Design Analysis. American Society of Mechanical Engineers paper $\mathrm{N}^{\circ} 82166$, Nantes, July 2-4

10. Chang M, Park SC (2008) Reverse engineering of a symmetric object. Computers and Industrial $\begin{array}{llll}\text { Engineering } & 55 & (2): & 311-320 .\end{array}$ doi:10.1016/j.cie.2007.12.015

11. Tuzikov AV, Sheynin SA, Vasiliev PV (2003) Computation of volume and surface body moments. Pattern Recognition 36(11): 2521-2529

12. Liu YS, Ramania K (2009) Robust principal axes determination for point-based shapes using least median of squares. Computer-Aided Design 41(4): 293-305

13. Soldea O, Elber G, Rivlin E (2002) Exact and efficient computation of moments of free-form surface and trivariate based geometry. ComputerAided Design 34: 529-539
14. Crisco J.J, McGovern RD (1997) Efficient calculation of mass moments of inertia for segmented homogeneous three-dimensional objects. Journal of Biomechanics 31(1) 97101.doi:10.1016/S0021-9290(97)00108-5

15. Lichter MD, Dubowsky S (2003) Estimation of state, shape, and inertial parameters of space objects from sequences of range images. Photonics Technologies for Robotics, Automation, and Manufacturing. International Society for Optics and Photonics194-205

16. Jones MW (1996) The Production of Volume Data from Triangular Meshes using Voxelisation. Computer Graphics Forum 15(5):311-318. doi: $10.1111 / 1467-8659.1550311$

17. Osty G (2002) Extraction de particularités géométrique sur données discrètes issues de la numérisation: partitionnement de grand nuage de points. PhD dissertation, Ecole Normale Supérieure de Cachan

18. Patil S, Ravi B (2005) Voxel-based representation, display and thickness analysis of intricate shapes. Ninth International Conference on Computer Aided Design and Computer Graphics (CAD-CG'05) 6, Hong Kong, December 7-10.doi: 10.1109/CADCG.2005.86

19. Zuquete-Guarato A, Quinsat Y, Mehdi-Souzani C, Lartigue C (2012) Détermination de la qualité de numérisationpour l'équilibrage de vilebrequins. $13^{\text {ème }}$ Colloque National AIP PRIMECA, Le Mont Dore, March 27-30

20. Mehdi-Souzani C, Quinsat Y, Lartigue C, Bourdet P (2016) A knowledge database of qualified digitizing systems for the selection of the best system according to the application. CIRP Journal of Manufacturing Science and Technology 13: 15-23

21. Lartigue C, Quinsat Y, Mehdi-Souzani C, ZuqueteGuarato A, Tabibian S (2014) Voxel-based path planning for 3D scanning of mechanical parts. Computer-Aided Design and Applications11(2): 220-227

22. Ticona EM, Guarato AZ, Braga SL (2015) Mathematical modeling and analysis of thermodynamic processes of an irreversible miller cycle working on a swing piston engine.23th $\mathrm{ABCM}$ International Congress of Mechanical Engineering COBEM2015, Rio de Janeiro, December 6-11 
23. Guarato AZ, Ticona EM, Braga SL (2015) Application of Atkinson / Miller cycle on a rotary internal combustion engine.23th ABCM International Congress of Mechanical Engineering COBEM2015, Rio de Janeiro, December 6-11

24. Guarato AZ, Ticona EM, Braga SL (2016) Development of a flex-fuel rotary engine with variable compression ratio. SAE Brasil 2016, São Paulo, October 25-27

25. Guarato AZ, Loja AC, Pereira LP, Braga SL, Trevilato TRB (2016) Qualification of a 3D structured light sensor for a reverse engineering application. Proc. SPIE 10151, Optics and Measurement International Conference, Liberec, October 11-14. doi: 10.1117/12.225760

26. Copying of free-forms from digitized data : The voxel space representation A. CONTRI, C. LARTIGUE, G. OSTY 3nd International Conference on Integrated Design and Manufacturing In Mechanical Engineering, IDMME'2000, Montréal (Canada), CDRom paper, 8 pages, May 2000 